\title{
Cardiovascular disease in pediatric patients under chronic peritoneal dialysis
}

\section{Compromiso cardiovascular en pacientes pediátricos en diálisis peritoneal crónica}

\author{
Paula Lehmann F. ${ }^{\mathrm{a}}$, Francisco Cano Sch. ${ }^{\mathrm{b}}$
}

aPediatric Nephrologist, Faculty of Medicine, Universidad Austral de Chile, Sub Department of Pediatrics, Valdivia Base Hospital.

${ }^{b}$ Division of Pediatric Nephrology, Luis Calvo Mackenna Childrens Hospital, Faculty of Medicine, University of Chile

Received: 2-3-2016; Accepted: 26-8-2016

\begin{abstract}
Peritoneal dialysis (PD) is the most common renal replacement therapy used in pediatric patients with end stage renal disease. This population has a mortality rate 1,000 times greater compare to pediatric population, mainly due to cardiovascular causes. Objective: To characterize pediatric patients on chronic PD in relation to dialysis and cardiovascular outcome. Patients and Methods: Cross sectional study. Patients in stable PD according to DOQI criteria were selected. Epidemiological, dialytic, biochemical and cardiovascular variables were registered. Left Ventricular Mass Index (LVMI) was calculated by height/age $\left(\mathrm{g} / \mathrm{m}^{2.7}\right)$. Left Ventricular Hypertrophy $(\mathrm{LVH})$ was diagnosed with $>38.6$ $\mathrm{g} / \mathrm{m}^{2.7}$, severe $\mathrm{LVH}>51 \mathrm{~g} / \mathrm{m}^{2.7}$. Data were analyzed using STATA 11.0. continuous variables using ANOVA test and categorical variables were analyzed using $\chi^{2}$ test or Fisher's exact test. Results: 21 patients, 11 males. Mean age $9.2 \pm 3.52$ years. The most frequent diagnosis was renal dysplasia (52\%). Residual and Peritoneal KtV were 0.8 and 1.9 respectively. Fifty-two percent of patients showed LVH, $91 \%$ in severe range. A significant relationship between ultrafiltration $/ \mathrm{m} 2$ and systolic blood pressure was depicted. Also a significant relationship between left ventricular mass index and hemoglobin $(\mathrm{p}<0.05)$ was founded. Conclusions: The majority of the population showed left ventricular hypertrophy -particularly severe LVH-, which confirms an increased CV risk in this population. Blood pressure and loss of ultrafiltration were founded to be correlated to LVH.
\end{abstract}

Keywords:

Chronic Kidney

Disease;

peritoneal dialysis;

left ventricular

Hypertrophy;

left ventricular mass

index

\section{Introduction}

Peritoneal dialysis (PD) is the most commonly used renal replacement modality in pediatric patients with terminal chronic kidney disease (CKD). The North American Pediatric Renal Trials and Collaborative Studies 2011 (NAPRTCS), showed that among 5,300 patients, 58\% used PD as renal replacement therapy $(\mathrm{RRT})^{1}$. In Chile, in 1994, 75\% of the pediatric patients on dialysis were on hemodialysis, while in 1999, 75\% were on peritoneal dialysis. These figures differ from the adult world, where the prevalence of hemodialysis (HD) patients at August 2008 was 13,613, compared with 594 patients in $\mathrm{DP}^{2}$. 
Children with CKD on dialysis have a significantly higher mortality rate than the rest of the pediatric population $^{3-5}$. The ANZDATA study reported that the mortality rate is 30 times higher than in pediatric patients without $\mathrm{CKD}^{6}$. Among the causes of mortality, cardiovascular disease corresponds to the $50 \%$ of the patients deaths on dialysis?

Cardiovascular disease is closely linked to volume overload, hypertension, and left ventricular hypertrophy (LVH). According to the International Pediatric Peritoneal Dialysis Network (IPPN $)^{8}$, the prevalence of $\mathrm{LVH}$ in children in PD reaches $40 \%$. From these, an adequate dialytic control achieves a regression of up to $50 \%$.

In pediatrics, the prevalence of $\mathrm{LVH}$ in children with end stage renal disease (CKD-5) is not well established. Mitsnefes M. et al (10) reported a figure of $75 \%$, and the IPPN registry showed that only $26 \%$ of PD patients had normal heart condition ${ }^{8}$. The NAPRTCS 2008 Registry showed that, from 4,000 patients on dialysis, $57 \%$ were with uncontrolled hypertension (>95\%), confirming that in patients with PD, hypertension and cardiac damage are most frequently associated with $\mathrm{LVH}^{7}$.

The definition of ventricular hypertrophy has been controversial because of factors affecting left ventricular mass (LVM), such as age, gender, surface and body composition. The most commonly used method for defining MVI in adults has been an allometric index that normalizes left ventricular mass to height (2.7), a variable called Left Ventricular Mass Index (LVMI). In adult uremic patients CKD-5, a significant relationship was found between a MVI $>51 \mathrm{~g} / \mathrm{m}^{2.7}$ and morbidity and mortality, defining this value as LVH. In children, a value of $38.6 \mathrm{~g} / \mathrm{m}^{2.7}$ corresponds to $\mathrm{p} 95$ of LVMI of the healthy pediatric population between 6 and 17 years old ${ }^{11}$. Khoury et $\mathrm{al}^{13}$ evaluated LVMI in 2,237 healthy and non-obese pediatric patients over 9 years of age, confirming that values $>40 \mathrm{~g} / \mathrm{m}^{2.7}$ in girls, and
$>45 \mathrm{~g} / \mathrm{m}^{2.7}$ in children, were above the percentile 95 . These authors emphasize that in children $<9$ years this index varies with age and therefore they suggest correcting LVMI for the size/age variable of the patient.

Until there is more evidence regarding the definition of LVH in the pediatric age, the cut-off values used in most studies correspond to those suggested by the fourth report on the diagnosis, evaluation, and treatment of hypertension in children and adolescents, from the US National Institutes of Health ${ }^{11}$.

The aim of the present study was to characterize a group of pediatric patients on chronic peritoneal dialysis from the cardiovascular point of view.

\section{Patients and Methods}

Cross-sectional, observational study. Pediatric patients with terminal chronic renal disease on controlled peritoneal dialysis at the Luis Calvo Mackenna Hospital between 2012 and 2014 were evaluated.

Inclusion criteria were considered patients in $\mathrm{PD}$ older than 4 months of life at the time of the cut, and at least 3 months of time in peritoneal dialysis. Exclusion criteria were antecedents of structural heart disease.

Anthropometric, clinical, epidemiological, dialytic, biochemical and echocardiographic variables were recorded.

\section{Anthropometric variables}

Weight and height, according to $Z$ score/age (ZT/E), $\mathrm{Z}$ weight/age (ZP/E), body mass index (BMI) calculated as weight in $\mathrm{kg}$ divided by height in square meters and area (ASC), calculated as the square root of weight in $\mathrm{kg}$ per size in $\mathrm{cm}$ divided by 3600 . Clinical variables: systolic and diastolic blood pressure (DBP) expressed in percentiles ( $>95$ or $<$ p95) according to Age, gender and height, according to reference (11). Biochemical parameters: Hemoglobin $(\mathrm{Hb})(\mathrm{mg} / \mathrm{dl})$,

Table 1. Study group characterization

\begin{tabular}{|c|c|c|c|c|c|c|c|c|c|}
\hline \multirow[t]{2}{*}{$\begin{array}{l}\text { Patients } \\
(\mathrm{n}=21) \\
\end{array}$} & \multicolumn{2}{|c|}{$\begin{array}{l}\text { Gender } \\
(\%)\end{array}$} & \multirow{2}{*}{\multicolumn{2}{|c|}{$\begin{array}{c}\begin{array}{c}\text { Age } \\
\text { (average years) }\end{array} \\
9.2 \pm 3.5\end{array}$}} & \multirow{2}{*}{\multicolumn{2}{|c|}{$\begin{array}{c}\begin{array}{c}\text { Time in PD } \\
\text { (average month) }\end{array} \\
32.4\end{array}$}} & \multirow{2}{*}{$\begin{array}{c}\begin{array}{c}\text { Anemia } \\
(\mathrm{Hb}<11 \mathrm{gr} / \mathrm{dl})\end{array} \\
\text { Yes } 33 \% \\
\text { No } 66 \%\end{array}$} & \multirow{2}{*}{$\begin{array}{c}\begin{array}{c}\mathrm{iPTH} \\
\text { (average } \mathrm{pg} / \mathrm{ml} \text { ) }\end{array} \\
347.5\end{array}$} & \multirow{2}{*}{$\frac{\begin{array}{c}\text { Ca*P } \\
\text { (average } \mathrm{mg} / \mathrm{dl} \text { ) }\end{array}}{58.7}$} \\
\hline & $\begin{array}{l}\text { Male } \\
\text { Female }\end{array}$ & $\begin{array}{l}48 \% \\
52 \%\end{array}$ & & & & & & & \\
\hline \multirow[t]{2}{*}{ Dialysis } & Mode & & D/P creat $_{2}$ & & D/D Gluc 2 & & $\mathrm{Kt} N$ perit & $\mathrm{Kt} N$ res & anuria \\
\hline & $\begin{array}{l}\text { NIPD } \\
\text { CCPD }\end{array}$ & $\begin{array}{l}72 \% \\
28 \%\end{array}$ & $\begin{array}{l}\text { High-high /average } \\
\text { Low-low /average }\end{array}$ & $\begin{array}{l}55 \% \\
45 \%\end{array}$ & $\begin{array}{l}\text { High-high /average } \\
\text { Low-low /average }\end{array}$ & $\begin{array}{l}90 \% \\
10 \%\end{array}$ & 1.9 & 0.91 & $52 \%$ \\
\hline Cardiovascular & $\mathrm{HBP}(>\mathrm{p} 95)$ & $48 \%$ & LVH (IMVI>p95) & $52 \%$ & LVMI $\left(\mathrm{g} / \mathrm{m}^{2,7}\right)$ & 48.43 & & & \\
\hline
\end{tabular}

PD: peritoneal Dialysis; iPTH: parathyroid hormone; Ca*P: calcium Phosphorus product; D/P creat ${ }_{2}$ : ratio of plasma / creatinine hour 2 ; D/D $\mathrm{Gluc}_{2}$ : ratio of dialysate glucose at hour 2 vs hour $0 ; \mathrm{Kt} / \mathrm{N}$ perit: peritoneal $\mathrm{Kt} / \mathrm{N} ; \mathrm{Kt} / \mathrm{N}$ res: residual $\mathrm{Kt} / \mathrm{N}$; HBP: high Blood Pressure; LVI: Left ventricular Hypertrophy; LVMI: Left Ventricula mass Index. 
vitamin D ( $\mathrm{pg} / \mathrm{ml})$, calcium $(\mathrm{Ca})(\mathrm{mg} / \mathrm{dl})$, Phosphorus (P) (mg/dl) (Vitros ${ }^{\circledR} 4600$ Chemistry System), parathyroid hormone $(\mathrm{Pg} / \mathrm{ml}$, Immunotopics, San Clemente, CA), FGF23 (pg/ml ELISA, Immunotopics, San Clemente, CA) and Klotho (pg/ml, ELISA, Cusabio, China) during the previous three months To echocardiography. Dialysis variables: dialysis volume/ cycle $\left(\mathrm{cc} / \mathrm{m}^{2}\right)$, dialysis type, peritoneal and residual dialysis dose (Kt V), ultrafiltration $\mathrm{ml} / 24 \mathrm{~h}(\mathrm{UF})$, water transport characteristics and solutes of the peritoneal membrane by PET $^{5}$.

A cardiovascular evaluation was performed by twodimensional color Doppler echocardiography performed up to 3 months before the cut. Echocardiography was performed by the same cardiologist at the Luis Calvo Mackenna Hospital. Systolic diameter, end diastolic diameter, interventricular septum thickness and posterior wall thickness were recorded. Left ventricular measurements were corrected for body surface area and expressed on a Z-score scale.

The MVI was calculated as the grams/height index 2.7, according to the Fourth Report of Hypertension in Children and Adolescents of the National High Blood Pressure Education Program Working Group11. HVI was defined as $>38.6 \mathrm{~g} / \mathrm{m}^{2.7}$, which corresponds to $\mathrm{p} 95$ of the LVMI for healthy children and adolescents between 6 and 17 years old. Severe LVH was defined as an MVI $>51 \mathrm{~g} / \mathrm{m}^{2.7}$, according to the criteria of the International Pediatric Peritoneal Dialysis Network (www. pedpd.org).

Statistical analysis of the data was performed using STATA 11.0 or SPS 15.0. Continuous variables were analyzed by ANOVA and categorical variables using $\chi^{2}$ or Fisher's Exact Test.

Consent and/or informed consent was requested to all participating patients. The study was approved by the Ethics and Human Research Committee of the Faculty of Medicine of the University of Chile.

\section{Results}

Twenty-one patients were included, 11 males, mean age $9.2 \pm 3.52$ years old. The mean weight and height were $28.3 \pm 9.8 \mathrm{~kg}$ and $124.8 \pm 18.5$ centimeters, with a $\mathrm{Z}$ average weight of -0.615 and $\mathrm{Z}$ average size of -1.92 . The average body surface area was $0.98 \pm$ $0.24 \mathrm{~m}^{2}$ (Table 1).

\section{General characteristics}

The most frequent diagnosis was renal dysplasia (11 patients, $52 \%$ ), followed by three patients with resistant cortical nephrotic syndrome and three patients with atypical hemolytic uremic syndrome. A patient with Alport's syndrome, one with renal polycystic di- sease, one with reflux nephropathy and one patient with an unrelated etiology.

\section{Dialytic features}

Patients were on automated peritoneal dialysis. The mean number of months on dialysis was 32.42 months. Eleven patients did not present residual diuresis, ten showed an average diuresis of $975 \mathrm{cc} / \mathrm{m}^{2} /$ day. The average body surface adjusted ultrafiltration (UF) was $573 \mathrm{cc} / \mathrm{m}^{2}$. The mean peritoneal Kt V (dialysis dose) was 1.9 and the total $\mathrm{Kt} \mathrm{V}$ (peritoneal plus residual) was 2.7 .

During 2 hours of peritoneal balance test, creatinine $\mathrm{D} / \mathrm{P}$ creatinine concentration (plasma creatinine hour 2) showed $45 \%$ of high average transporters $(\mathrm{n}=9) 35 \%$ low average $(\mathrm{n}=7)$ a $10 \%$ High transporter $(n=2)$, and $10 \%$ under carrier $(n=2) . D / D$ glucose (ratio of dialysate glucose at hour 2 vs hour $0)$ showed $50 \%$ of medium $(\mathrm{n}=1), 40 \%$ high $(\mathrm{n}=8)$ and $10 \%$ average low transporter $(\mathrm{N}=2)$. A significant relationship was found between total $\mathrm{KtV}$ and ultrafiltration, and between $\mathrm{D} / \mathrm{P}$ creatinine and $\mathrm{D} / \mathrm{D}$ glucose $(\mathrm{p}<0.05)($ Table 1$)$.

\section{Cardiovascular characteristics}

A value of $\mathrm{PA}>\mathrm{p} 95$ was recorded in ten patients $(48 \%)$ versus eleven $(52 \%)$ with PA $<$ p95. The Echocardiography analysis showed LVH in 11 patients (52\%), of which $91 \%$ corresponded to severe grade. (Table 1).

There was a statistically significant relationship between blood pressure and body surface area, as well as between body surface area adjusted $\mathrm{cc} / \mathrm{m}^{2}$ and SBP, with a similar trend, although without statistical significance with DBP. A significant relationship was found between LVMI and hemoglobin, and between LVMI and calcemia $(\mathrm{p}<0.05)$.

The average phosphorus calcium product was 58.70 and the $\mathrm{iPTH}$ was $347.5 \mathrm{pg} / \mathrm{dl}$. Only one patient presented $\mathrm{PTH}<100$, and ten patients showed values $>300 \mathrm{pg} / \mathrm{dl}$. The relationship between iPTH and FGF23 was significant $(\mathrm{p}<0.05)$. The mean values of FGF23 and Klotho were $225.7 \pm 310$ and $126 \pm 20$ pg/ $\mathrm{ml}$, respectively. Seven patients had anemia at the time of the study, with an average $\mathrm{Hb}$ of $11.13 \mathrm{mg} / \mathrm{dl}$ in the total of the patients. The mean ferritin was $216 \mathrm{mg} / \mathrm{dl}$.

\section{Discussion}

LVH has a high prevalence in the pediatric population CKD-510. Associated factors include hypertension, body mass index, type of peritoneal dialysis applied, anemia and hyperparathyroidism, as well as the etiology of CKD other than renal dysplasia ${ }^{8,14,15}$. 
Among the protective factors are the management of adequate blood pressure, maintaining a euvolemic state, correction of metabolic alterations characteristic of the terminal CKD (calcium phosphorus and base acid metabolism), correction of anemia and management of uremia.

LVH has been associated with 3 groups of factors: with post-load, such as increased systemic arterial resistance, elevated blood pressure and vascular compliance; with preload, such as intravascular volume expansion and anemia; And other independent factors, such as microinflammation and oxidative stress 9 .

Various cutoff values have been used for the diagnosis of left ventricular hypertrophy. The most commonly used are adjusted according to the size of the patient ${ }^{9}$. HVI is defined as the LVMI that exceeds p95 for gender and age of normal children and adolescents (Khoury et al) ${ }^{13}$. The International Pediatric Peritoneal Dialysis Network (IPPN) is based on these same recommendations. Values previously estimated by Mitsnefes with a more limited number of patients, found that LVMI was $68-73 \%$ in patients in $\mathrm{PD}^{7}$ and $80-85 \%$ in patients in $\mathrm{HD}^{7,16}$. The collaborative study published by the IPPN found that the use of height-associated LVMI provides the most prevailing prevalence of $\mathrm{LVH}$ in patients with $\mathrm{PD}^{8}$.

In this study we confirmed the severe cardiovascular compromise of pediatric patients on chronic peritoneal dialysis. Its impact on the medium and long-term survival is paramount, which confirms the importance of a routine monitoring in cardiovascular patients.

Among the objectives in an individualized PD are an adequate UF that prevents hypervolemia, one of its clinical signs being systolic arterial hypertension. Hypertension in PD patients can be managed with volume restriction, leading the patient to its dry weight ${ }^{18}$. Blood purification of solutes should be achieved, not only limited to urea ${ }^{4,19,20}$. In our study, a significant correlation was found between UF and SBP. BP was measured in isolation, without continuous ambulatory blood pressure monitoring. In the study by Mistefens et $\mathrm{al}^{16}$ it was determined that nocturnal values of SBP greater than $\mathrm{p} 95$ had an independent association with the increase in LVMI. In the study by Bircan et $\mathrm{al}^{18}$, it was shown that random values of PAS $>95$ had a sensitivity of $52 \%$ and a negative predictive value of $41 \%$ for predicting $\mathrm{LVH}$, not in agreement with the criteria of Sorof et $\mathrm{al}^{21}$, which reports a prevalence of HIV of $47 \%$ in patients with PAS values greater than $50 \%$.

The risk of developing LVH is 2 times higher in children with systolic hypertension. In the follow-up of patients in the IPPN collaborative study, SBP was $7 \mathrm{mmHg}$ higher in patients who developed or maintained LVH than in those who returned to $\mathrm{LVH}$ and achieved normal LVMI values. Studies have shown that decreasing volume overload and blood pressure over a period of 6 months leads to an improvement in left ventricular mass index values in children with LVH in $\mathrm{PD}^{17}$.

From the dialysis dose point of view, the $\mathrm{KtV}$ is the recommended parameter to evaluate the adequacy of the procedure ${ }^{4,19,22}$. The total $\mathrm{KtV}(\mathrm{Kt} / \mathrm{Vt})$, expresses the sum of the weekly urea clearance according to the scheduled dialysis indication, called peritoneal Kt/V $(\mathrm{Kt} / \mathrm{Vp})$, plus the residual renal function contribution that still maintains the patient through diuresis, called residual $\mathrm{Kt} / \mathrm{V}(\mathrm{Kt} / \mathrm{Vr})^{23}$. In our study, a significant relationship was found between total $\mathrm{KtV}$ and total UF. $\mathrm{LVH}$ has been inversely related to residual $\mathrm{KtV}$, with higher LVH in anuric patients than in patients with residual diuresis ${ }^{24,25}$. The CANUSA study showed that each $250 \mathrm{ml}$ of urine shows a $36 \%$ decrease in overall mortality ${ }^{26,27}$. Patients with residual renal function have better cardiovascular and metabolic profiles than anuric patients; they have less malnutrition, less hypertension, less ventricular hypertrophy, less anemia and lower calcium phosphorus product, with less vascular calcification. All this leads to a better cardiovascular survival in these patients ${ }^{28}$. In our study, there was no significant correlation between $\mathrm{LVH}$ and patients without residual diuresis, which may be due to the limited number of anuric patients in the study group. Approximately, a 50\% had a KtVr greater than 0.1 and in six of them the $\mathrm{KtVr}$ was greater than the $\mathrm{KtVp}$. Wang et al showed that residual renal function is an important independent predictor of LVH and one of the most important factors in adult patient mortality in $\mathrm{PD}^{24}$.

In patients on dialysis, LVH has been demonstrated without arterial hypertension, which implies that there are other mechanisms involved in the development of $\mathrm{LVH}$ in patients in chronic renal replacement therapies $^{7}$. In this sense, hemoglobin values below $11 \mathrm{mg} / \mathrm{dl}$ have been associated with mortality and LVH in children on dialysis ${ }^{17}$. Anemia leads to an increase in left ventricular size and ejection volume, possibly increasing cardiac adaptations to volume changes with dialysis ${ }^{7} .33 \%$ of patients in our study had anemia, with a significant relationship between hemoglobin and total $\mathrm{KtV}$, as well as left ventricular mass index.

There is an observed relationship between anomalies in mineral bone metabolism and $\mathrm{LVH}^{29,30}$. PTH has trophic effects on cardiac myocytes by stimulating cardiac fibroblasts and intramyocardial arterial wall thickness, resulting in LVH and intramyocardial fibrosis ${ }^{31,32}$. With Cutoff values of $200 \mathrm{ng} / \mathrm{ml}$ of PTH have been shown to be at a $73 \%$ risk of $\mathrm{LVH}$, suggesting that PTH cardiotropic actions already appear with moderate values of hyperparathyroidism ${ }^{33,34}$. On the other hand, vitamin $\mathrm{D}$ deficiency can activate the $\mathrm{Re}$ - 
nin Angiotensin Aldosterone system, and active vitamin D supplementation can cause regression of LVH and/or fibrosis. Calcitriol reduces cardiac fibrosis and microvascular remodeling in experimental models of renal failure ${ }^{41}$.

The fibroblast growth factor 23 (FGF 23), is a phosphaturic hormone that reduces the activity of 1 renal alpha hydroxylase and also the renal synthesis of 1.25 hydroxy vitamin $\mathrm{D}$, playing a central role in the maintenance of step homeostasis mineral Early stages of the ERC. Different studies, as in this experience, have shown a significant elevation of FGF23 in terminal $\mathrm{CKD}^{35,36}$. There are recent experiences linking cardiovascular involvement with plasma levels of FGF 23 in children. Seeherunvong et al demonstrated a strong association between high concentrations of FGF23 and elevated LVMI and that high values of FGF23 are associated with LVH in children and adolescents in HD. They did not find a relationship between FGF23 values and dialysis time, suggesting that the association between LVH and FGF23 could be evident in patients at the onset of $\mathrm{HD}$, showing that the relationship would be present prior to the initiation of dialytic therapy ${ }^{37}$.

The FGF 23 of our group was only obtained in twelve of our patients, corresponding to $62 \%$ of the group. We did not demonstrate an association between FGF23 and LVH values, probably due to the limited number of patients in whom FGF levels were available ${ }^{23}$. In patients with CKD, FGF23 concentrations progressively increase, as renal function decreases to help maintain serum phosphorus within normal ${ }^{38}$, which can lead to very high values of FGF23 correlated with the plasma phosphorus of dialysis patients ${ }^{39}$. Conversely, terminal ERC is considered to be a Klotho deficit state, which has also been evidenced by the values obtained in a subgroup of our patients. Despite not finding a significant association between these 2 hormones of mineral metabolism and LVH, we did observe a significant association between PTHi and FGF values ${ }^{23}$.

\section{Conclusion}

In this study, values of left ventricular hypertrophy were found to be greater than $50 \%$, corresponding mostly to severe $\mathrm{LVH}$, which evidences the severe cardiovascular involvement of this population. As in this study, the relationship between blood pressure and ultrafiltration have been previously objectified as causal factors of LVH. The importance of cardiovascular monitoring in pediatric patients on dialysis and adjustment of individualized dialysis is reinforced in order to improve the quality of life and survival of these patients.

\section{Ethical Responsibilities}

Human Beings and animals protection: Disclosure the authors state that the procedures were followed according to the Declaration of Helsinki and the World Medical Association regarding human experimentation developed for the medical community.

Data confidentiality: The authors state that they have followed the protocols of their Center and Local regulations on the publication of patient data.

Rights to privacy and informed consent: The authors have obtained the informed consent of the patients and/or subjects referred to in the article. This document is in the possession of the correspondence author.

\section{Financial Disclosure}

Authors state that no economic support has been associated with the present study.

\section{Conflicts of Interest}

Authors state that any conflict of interest exists regards the present study. 


\section{References}

1. North American Pediatric Renal Trials and Collaborative Studies. NAPRTCS 2011. Annual Dialysis Report.

2. Ministerio de salud. Guía clínica diálisis peritoneal. Santiago: Minsal, 2010.

3. Watanabe A, Lanzarini VV, Filho UD, Koch VH. Comparative role of PET and $\mathrm{Kt} / \mathrm{V}$ determination in pediatric chronic peritoneal dialysis. Int J Artif Organs 2012;35(3):199-207.

4. Fischbach M, Bradley A. Warady. Peritoneal dialysis prescription in children: bedside principles for optimal practice. Pediatr Nephrol 2009;24:163342.

5. Schmitt CP, Zaloszyc A, Schaefer B, Fischbach M. Review Article: Peritoneal Dialysis Tailored to Pediatric Needs. International Journal of Nephrology. Received 17 February 2011; Accepted 29 March 2011.

6. Shroff R, Ledermann S. Long-term outcome of chronic dialysis in children. Pediatr Nephrol 2009;24:463-44.

7. MItsnefes MM, Daniels SR, Schwartz SM, Meyer RA, Khoury P, Strife CF. Severe left ventricular hypertrophy in pediatric dialysis: prevalence and predictors. Pediatr Nephrol 2000;14(10-11):898-90.

8. Bakkaloglu SA, Borzych D, Soo Ha I, et al. Cardiac geometry in children receiving chronic peritoneal dialysis: findings from de International pediatric Peritoneal Dialysis network (IPPN) register. Clin J Am Nephrol 2011; 6(8):1926-33.

9. Glassock R, Pecoits-Filho R, Barberato S. Left ventricular mass in Chronic Kidney Disease and ESRD. Clin J Am Soc Nephrol 2009;4:S79-S 91.

10. Mistnefes M, Stablein D. Hypertension in pediatric patients on long-term dialysis: a report of the North American Pediatric Renal Transplant Cooperative Study (NAPRTCS). Am J Kidney Dis. 2005; 45(2):309-15.

11. National High Blood Pressure Education Program Working Group on High Blood Pressure in Children and Adolescents: The fourth report on the diagnosis, evaluation and treatment of high blood pressure in children and adolescents. Pediatrics 2004;114: 555-76.

12. Foster BJ, Mackie AS, Mitsnefes M, Ali H, Mamber S, Colan SD. A novel method of expressing left ventricular mass relative to body size in children. Circulation. 2008;117(21):2769-75.

13. Khoury PR, Mitsnefes M, Daniels SR, Kimball TR. Age-specific reference intervals for indexed left ventricular mass in children.J Am Soc Echocardiogr. 2009;22(6):709-14.

14. Mitsnefes MM. CV complications of pediatric chronic kidney disease. Pediatr
Nephrol 2008;23: 27-39.

15. Bakkaloglu SA, Saygili A, Sever L, et al. Assessment of cardiovas- cular risk in paediatric peritoneal dialysis patients: A Turkish Pediat- ric Peritoneal Dialysis Study Group (TUPEPD) report. Nephrol Dial Transplant 2009;24: 3525-32.

16. Mitstnefes MM, Barletta GM, Dresner IG, et al. Severe cardiac hypertrophy and long-term dialysis: The Midwest Pediatric Nephrology Consortium Study. Pediatr Nephrol 2006;21:1167-70.

17. Mahan J, Patel H. Recent advances in pediatric dialysis: a revew of selected articles. Pedatr nephrol 2008;23:1737-47.

18. Bircan Z. Predictors of left ventricular hypertrophy in children on chronic peritoneal dialysis. Pediatr Nephrol 2010;25:1311-8.

19. Fischbach M, Stefanidis CJ, Watson AR, European Paediatric Peritoneal Dialysis Working Group. Guidelines by an ad hoc European committee on adequacy of the paediatric peritoneal dialysis prescription. Nephrol Dial Transplant 2002;17:380-5.

20. Fischbach M, Lahlou A, Eyer D, Desprez P, Geisert J. Determination of individual ultrafiltration time (APEX) and purification phosphate time by peritoneal equilibration test. Application to individual peritoneal dialysis modality prescription in children. Perit Dial Int 1996;16(Suppl 1):S557-60.

21. Sorof JM, Cardwell G, Franco K, Portman RJ. Ambulatory blood pressure and left ventricular mass index in hypertensive children. Hypertension 2002;39:903-8.

22. Durand PY, Freida P, Issad B, Chanliau J. How to reach optimal creatinine clearances in automated peritoneal dialysis. Perit Dial Int 1996;16(Suppl 1):S167-70.

23. National Kidney Foundation. K/DOQUI clinical practice recomendation for 2006 updates: hemodialysis adecuacy, peritoneal dialysis adecuacy and vascular Access. Am j Kidney Dis. 2006; 48 (Suppl 1): S1-S322.

24. Wang AY, Wang M, Woo J, et al. Inflammation, residual kidney function, and cardiac hypertrophy are interrelated and combine adversely to en- hance mortality and cardiovascular death risk of peritoneal dialysis patients. J Am Soc Nephrol 2004;15: 2186-94.

25. Wang AY, Wang M, Woo J, et al. A novelassociation between residual renal function and lef ventricular hypertrophy in peritoneal dialysis patients. Kidney Int 2002; 62(2):639-47.

26. Rojo A, Azócar M, Ibacache MJ, et al. The
MiniPET in peditric peritoneal dialysis. A useful tool to predict volumen overload? Pediatr Nephrol 2013;28:1121-6.

27. Bargman JM1, Thorpe KE, Churchill DN; CANUSA Peritoneal Dialysis Study Group. Relativecontribution of residual renal function and peritoneal clearence to adecuacy of dialysis: a reanalysis of the CANUSA study. J Am Soc Nephrol. 2001;12(10):2158-62.

28. Wang AY. The John F. Maher Award Recipient Lecture 2006. The "heart" of peritoneal dialysis: residual renal function. Perit Dial Int. 2007;27(2):116-24.

29. Randon RB, Rohde LE, Comerlato L, Ribeiro JP, Manfro RC. The role of secondary hyperparathyroidism in left ventricular hypertrophy of patients under chronic hemodialysis. Braz J Med Biol Res 2005;38:1409-16.

30. Mitsnefes MM, Kimball TR, Kartal J, et al. Cardiac and vascular adaptation in pe- diatric patients with chronic kidney disease: Role of calcium- phosphorus metabolism. J Am Soc Nephrol 2005;16: 2796-803.

31. Schlüter KD, Piper HM. Trophic effects of catecholamines and parathyroid hormone on adult ventricular cardiomyocytes. Am J Physiol. 1992; 263(6 Pt 2):H1739-46.

32. Amann K, Ritz E, Wiest G, Klaus G, Mall G. A role of parathyroid hormone for the activation of cardiac fibroblasts in uremia. J Am Soc Nephrol 1994;4: 1814-9.

33. Saleh FN, Schirmer H, Sundsfjord J, Jorde R. Parathyroid hor- mone and left ventricular hypertrophy. Eur Heart J 2003;24: 2054-60.

34. Iqbal A, Jorde R, Lunde P, Sundsfjord J, Rasmussen K. Left ventricular dysfunction in subjects with mild secondary hyperparathyroidism detected with pulsed wave tissue Doppler echocardiography. Cardiology 2006; 105:1-8.

35. Gutiérrez OM, Mannstadt M, Isakova $\mathrm{T}$, et al. Fibroblast growth factor 23 and mortality among patients undergoing hemodialysis. N Engl J Med 2008 359:584-92.

36. Wesseling-Perry K, Pereira RC, Wang $\mathrm{H}$, et al. Relationship between plasma fibroblast growth factor-23 concentration and bone mineralization in children with renal failure on peritoneal dialysis. J Clin Endocrinol Metab 2009;94:511-7.

37. Seeherunvong W, Abitbol CL, Chandar J, Rusconi P, Zilleruelo GE, Freundlich M. Fibroblast growth factor 23 and left ventricular hypertrophy in children on dialysis. Pediatr Nephrol (2012) 27: 2129-36.

38. Gutiérrez O, Isakova $\mathrm{T}$, Rhee $\mathrm{E}$, et al. Fibroblast growth factor-23 mitigates hyperphosphatemia but accentuates 
calcitriol deficiency in chronic kidney disease. J Am Soc Nephrol 2005;16: 2205-15.

39. Isakova $\mathrm{T}$, Xie $\mathrm{H}$, Yang $\mathrm{W}$, et al. Fibroblast growth factor 23 and risks of mortality and end-stage renal disease in patients with chronic kidney disease. JAMA 2011;305:2432-2439
40. Seifert ME, de Las Fuentes L, Ginsberg C, et al. Left ventricular mass progression despite stable blood pressure and kidney function in stage 3 chronic kidney disease. Am J Nephrol. 2014;39:392-9.

41. Freundlich M, Li YC, Quiroz Y, et al. Paricalcitol downregulates myocardial renin-angiotensin and fibroblast growth factor expression and attenuates cardiac hypertrophy in uremic rats. Am J

Hypertens. 2014; 27(5): 720-6.

42. Devereux RB, Alonso DR, Lutas EM, et al. Echocardiographic assessment of left ventricular hypertrophy: Comparison to necropsy findings. Am J Cardiol 1986;57: 450-8. 